\title{
A matching-to-sample procedure for the study of visual perception and concept formation in infrahumans: $A$ test in the pigeon
}

\author{
FRED ALTMAN* and SUZANNE P. MILLER \\ University of Kentucky, Lexington, Kentucky 40506
}

\begin{abstract}
An af zaratus suitable for conducting simultaneous or delayed matching-to-sample experiments with as many as eight comparison stimuli is described. Data are presented which indicate that pigeons are capable of performing such a task. It is concluded that the simultaneous matching-to-sample procedure with more than two comparison stimuli is an efficient means of obtaining information about discrimination capacity and that this procedure may be suitable for studying visual perception in infrahuman Ss.
\end{abstract}

This study was undertaken to develop and test a methodology for the investigation of visual space perception and concept formation in infrahuman Ss. For such investigations, it is often necessary for Ss to locate stimuli on one or more dimensions selected by the $E$, e.g., Ss may be required to indicate the perceived size and distance of a stimulus. With human Ss, such stimulus characteristic identification is generally easily obtained with verbal instructions and verbal or nonverbal responses; with infrahuman Ss, however, the task is more difficult. The $E$ must find a means of communicating to the Ss the dimensions in which he is interested, and he must provide a means by which the Ss can indicate the perceived location of the stimulus on each dimension. In order to minimize confounding effects, the method should not involve a heavy memory load or require a highly complex response. The method should also be efficient, i.e., each response or each unit of time should yield as much information about the location of a stimulus as practical.

The only presently available experimental paradigm which seems to meet these requirements is "matching to sample." The Malotts have used a matching-type design with a go/no-go response to study perceptual phenomena (Malott \& Malott, 1970). With the matching-to-sample procedure, a dimension can be specified by varying the comparison stimuli along that dimension only.

To be certain that responding is under the control of the variable dimension, it must be shown that the Ss use this dimension and that they discriminate it from other dimensions. Matching-to-sample behavior, when the sample and matching comparison stimuli are identical, implies that the Ss use the variable dimension. However, it does not provide evidence that the Ss discriminate the variable dimension from other dimensions. Rather, they

* Present address: National Institute on Drug Abuse, Room 740G Rockwall Building, 11400 Rockville Pike, Rockville, Md. 20857 . may be making an identity match; matching on the basis of the sameness of all the stimulus attributes instead of only the sameness of the attribute which lies on the variable dimension.

Matching-to-sample behavior, when the sample stimulus differs from the matching comparison stimulus on at least one dimension, is probably the simplest way for the Ss to indicate that they discriminate the variable dimension from other dimensions. For example, to test a S's size discrimination, the comparison stimuli would have to be a set of stimuli varying in size but identical in all other dimensions (e.g., shape and color). The sample stimuli must also be a set of stimuli varying in size, and they could be the same as the comparison stimuli on the other dimensions as well. Both the comparison and sample stimuli could be composed of red circles. However, this test would not indicate whether the $S$ was matching on the basis of identity or on the basis of the size dimension. To test whether a $S$ is discriminating the size dimension, the sample stimuli must differ from the comparison stimuli on at least one dimension other than size. If the comparison stimuli are red circles, then the sample stimuli might be green circles.

The dimension along which the comparison stimuli vary can be changed between trials, making it possible to localize one stimulus on more than one dimension. Further, the sample and comparison stimuli can be presented simultaneously, minimizing the memory load. (Absolute judgments can also be obtained by using a delayed matching design.) With the matching-to-sample paradigm, responses can be made directly to the appropriate comparison stimulus, thus eliminating the need for more complex coding of the response information. Finally, matching to sample is highly efficient, since a single response can localize the stimulus along a dimension. The degree of localization is limited only by the number and the range of the comparison stimuli.

Although two comparison stimuli are enough to identify a linear dimension, a greater number of comparison stimuli may not only improve the efficiency of obtaining information, but may also improve communication with the Ss about the dimension. The improved communication would occur when the dimension along which the comparison stimuli vary is difficult to detect and the addition of comparison stimuli make the variable dimension more apparent.

In order to use the simultaneous matching-to-sample procedure to locate stimuli on dimensions, it must first be established that the Ss are capable of performing a matching task with a suitable number and configuration of stimuli. There are a limited number of studies using 


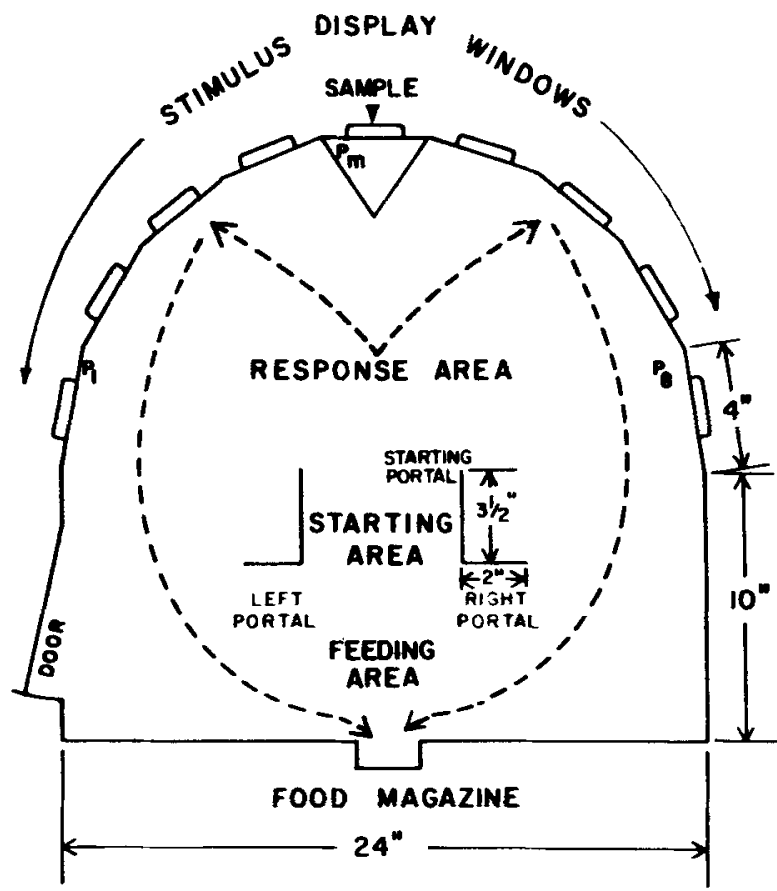

Fig. 1. Plan of apparatus. Dotted lines indicate the general response paths of the pigeons.

more than two comparison stimuli; the largest number of comparison stimuli reported is six, and that was on a sequential matching task (Cohen, 1969). The present study was conducted to determine whether or not pigeons were capable of performing on a simultaneous matching task with as many as eight comparison stimuli (three bits of information transmission per response). With this number, a reasonable amount of information (three bits) can be obtained per response; eight choices approach the limit of absolute judgment (Miller, 1956); and the addition of comparison stimuli is costly and becomes progressively more difficult. Specifically, the matching task was designed for subsequent use in the study of visual space perception.

\section{METHOD}

Subjects

The Ss were four male Carneaux pigeons between 2 and 3 years old. All $S$ s had been used several months prior to this experiment in a brightness discrimination study. The birds were maintained at approximately $75 \%$ of their free-feeding weights.

\section{Apparatus}

The apparatus provided nine fairly large stimulus display windows in a configuration which could be used to study size distance invariance and other aspects of visual space perception.

The shape of the experimental chamber was that of a rectangle with one side bowed outward in a semicircle (Fig. 1). The semicircular side was constructed of nine stimulus panels. On each of these panels, there was a window behind which the stimulus display was located (Fig. 2). The middle panel $\left(\mathrm{P}_{\mathrm{m}}\right)$ contained the sample stimulus display ( $\mathrm{Ss}$ ), and the eight side panels $\left(\mathrm{P}_{1}-\mathrm{P}_{\mathrm{s}}\right.$ sequentially starting from the left side and omitting $\mathrm{P}_{\mathrm{m}}$ ) contained the comparison stimulus displays $\left(\mathrm{S}_{\mathbf{c}}\right)$. A triangular block extended out from the middle panel to discourage pecking at the sample window. Opposite the middle panel $\left(P_{\mathbf{m}}\right)$, equidistant from all the stimulus panels, was the end of a passage. The other end of this passage led to the food magazine, which was located in the center of the rear wall. This passage served as the starting area for a trial. Above the food magazine was a window, through which the E could observe. A Foringer white noise generator directed a masking noise into the chamber. On the outside of the chamber, each stimulus display window was individually enclosed and illuminated by an incandescent bulb located in the enclosure but below the window itself. The inside of each enclosure was painted a glossy white to provide maximum reflectance.

A Masonite rectangle served as a stimulus insert and was located against the chamber wall in each comparison stimulus enclosure. They could be removed and replaced without removing the back of the enclosure. In the chamber, the inserts produced a black field behind the stimulus windows. When the enclosures were illuminated, holes in the inserts appeared as white stimuli on a black field (Fig. 2). All the sample stimuli were cut into an 11-in. Masonite disk, which could be rotated through the sample stimulus display window enclosure in order to change the stimulus. Thus, with the same size and shape hole in the sample stimulus display window and a comparison stimulus display window, the comparison and sample stimuli were identical. The stimuli were circles varying in diameter in 1/8-in. increments. They were numbered sequentially from largest, $S_{1}\left(\left[1 / 2\right.\right.$ in.) to smallest, $S_{8}(5 / 8$ in.).

Varying the stimulus size in this way affects not only the size, but also the total light available. The Ss could match on the basis of either variable or some combination of them. For this study, the specific dimension or dimensions used is irrelevant, although in future studies, it will be necessary to specify the dimension.

Trials were initiated by a manual switch. At the start of a trial, the sample stimulus was illuminated first, and after a 1 -sec interval (comparison stimulus delay) those comparison stimuli which were switched to a conditional on state were illuminated. Since the windows did not contain a provision for response sensing, the response sequences were also initiated by a manual switch; in the correct response sequence, the stimulus lights were extinguished and the food magazine light actuated for a preset interval (reinforcement period). During the reinforcement period, the light over the feeding area was also brightened. In the event of an incorrect response, both the chamber lights and the stimulus lights were extinguished for a period of $60 \mathrm{sec}$.

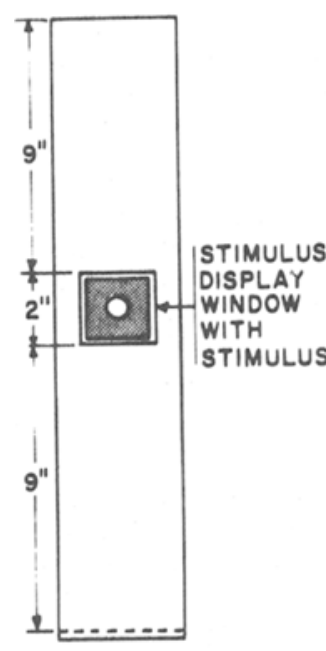

A.

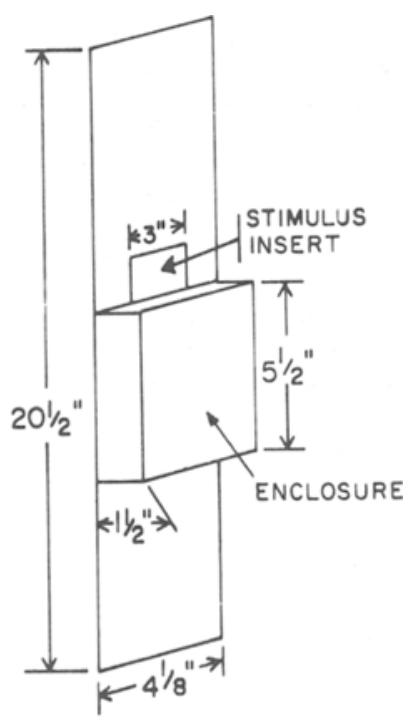

8
Fig. 2. Diagrams of a stimulus panel. (A) View from inside chamber. (B) View from outside chamber. 

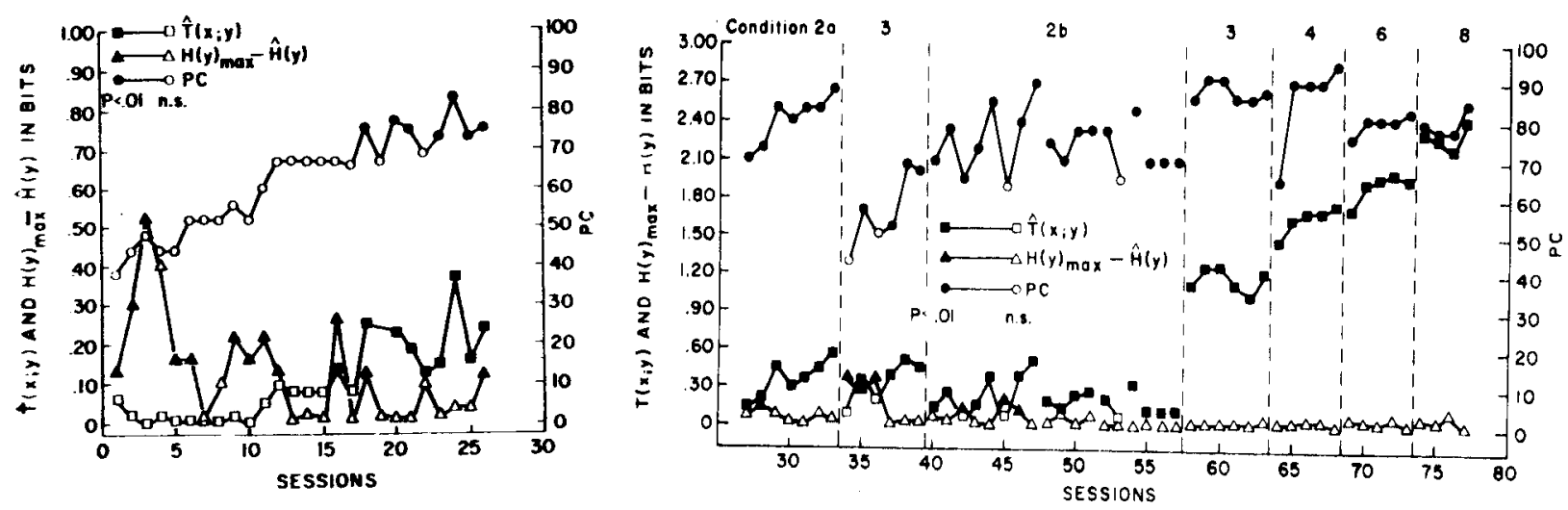

Fig. 3. The amount of information about the sample stimulus transmitted by the responses, $T(x ; y)$; the position preference, $\mathrm{H}(\mathrm{y})_{\max }-\hat{\mathrm{H}}(\mathrm{y})$; and the percentage of correct responses, PC, in each training session for $\mathrm{S} 11$. (A) Condition 1 . (B) Conditions 2-8.
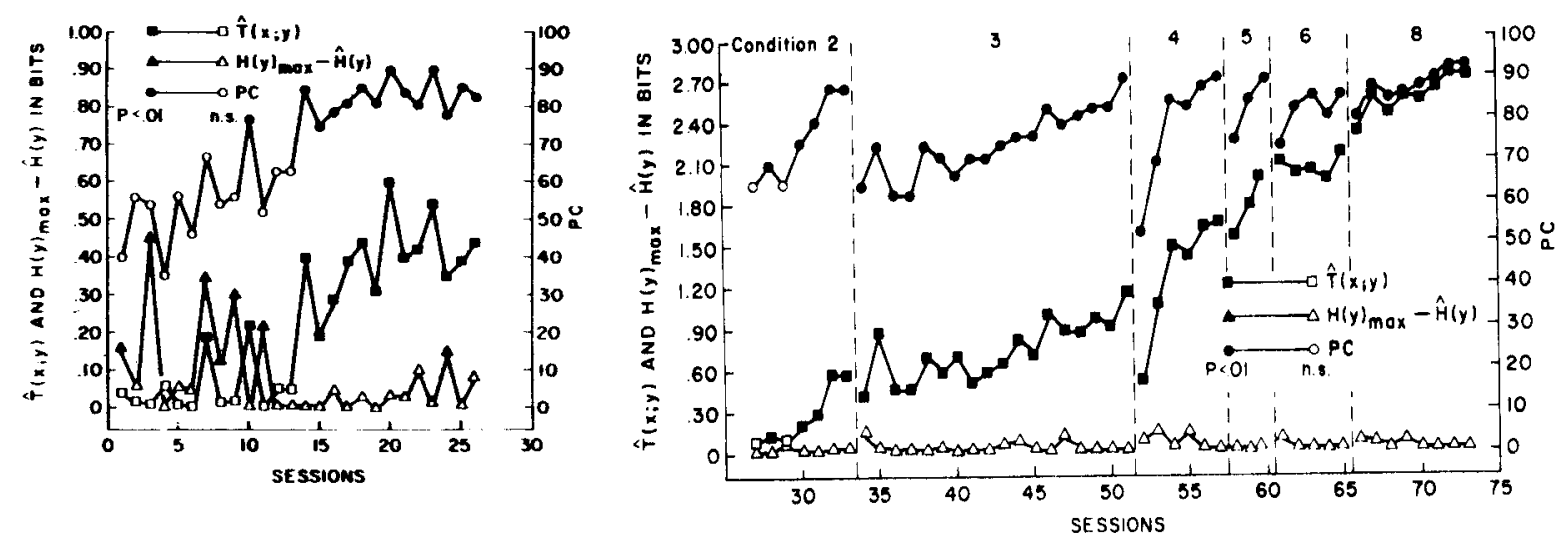

Fig. 4. The amount of information about the sample stimulus transmitted by the responses, $T(x ; y)$; the position preference, $\mathrm{H}(\mathrm{y})_{\max }-\hat{\mathbf{H}}(\mathrm{y})$; and the percentage of correct responses, $\mathrm{PC}$, in each training session for S 12 . (A) Condition 1 . (B) Conditions 2-8.

transforming their values to chi square (Attneave, 1959). These measures were used to obtain an indication of the difficulty of the task and of the response distribution, neither of which are provided by the percentage correct score.

Figures 3, 4, 5, and 6 show the progress of the Ss in matching training. It can be seen from these figures that Ss 11,12 , and 14 acquired each of the training tasks fairly rapidly, while S 13 failed to master even the first condition. It is also evident that the additional training with all adjacent pairs of stimuli did not noticeably improve the performance of $\mathrm{S} 11$ in the subsequent conditions. As indicated by the $\mathrm{H}(\mathrm{y})_{\max }-\hat{\mathrm{H}}(\mathrm{y})$ measure in the first condition, all Ss displayed a position preference, but all except S 13 lost this preference between the 12th and 19th sessions. S 13 never abandoned his preference. Because Bird 13 did not attain a satisfactory level of performance in this condition, he was dropped from the experiment. Position preference was not a problem in any of the subsequent conditions, although there were a few sessions in which $\mathrm{H}(\mathrm{y})_{\max }-\hat{\mathrm{H}}(\mathrm{y})$ was relatively high.

Performance during all of the test conditions (Test Conditions 2, 4, 6, 8, and 8-reversed) was well above the chance level; only one $\mathrm{S}$ in one condition made less than $90 \%$ correct responses (Table 2 ).

It is clear from these results that the pigeons are capable of performing a simultaneous matching task with eight simultaneously presented comparison stimuli.

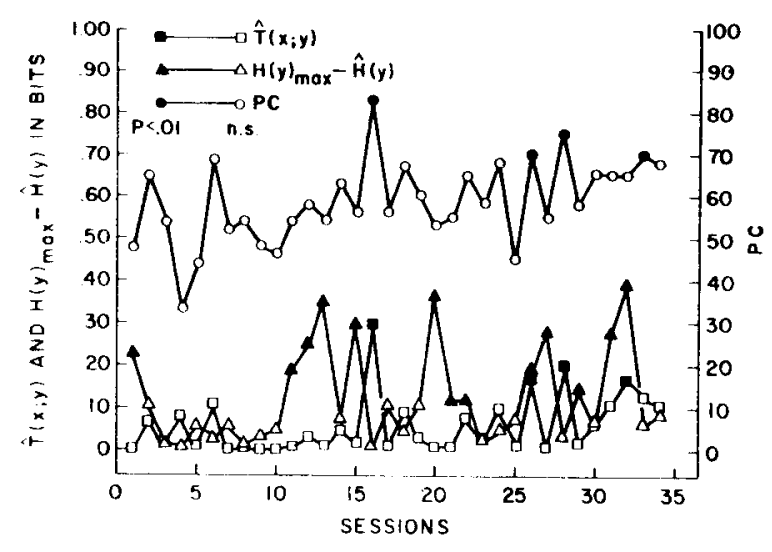

Fig. 5. The amount of information about the sample stimulus transmitted by the responses, $T(x ; y)$; the position preference, $H(y)_{\max }-\mathbf{H}(y)$; and the percentage of correct responses, PC, in each training session for $\mathrm{S} 13$ in Condition 1 . 

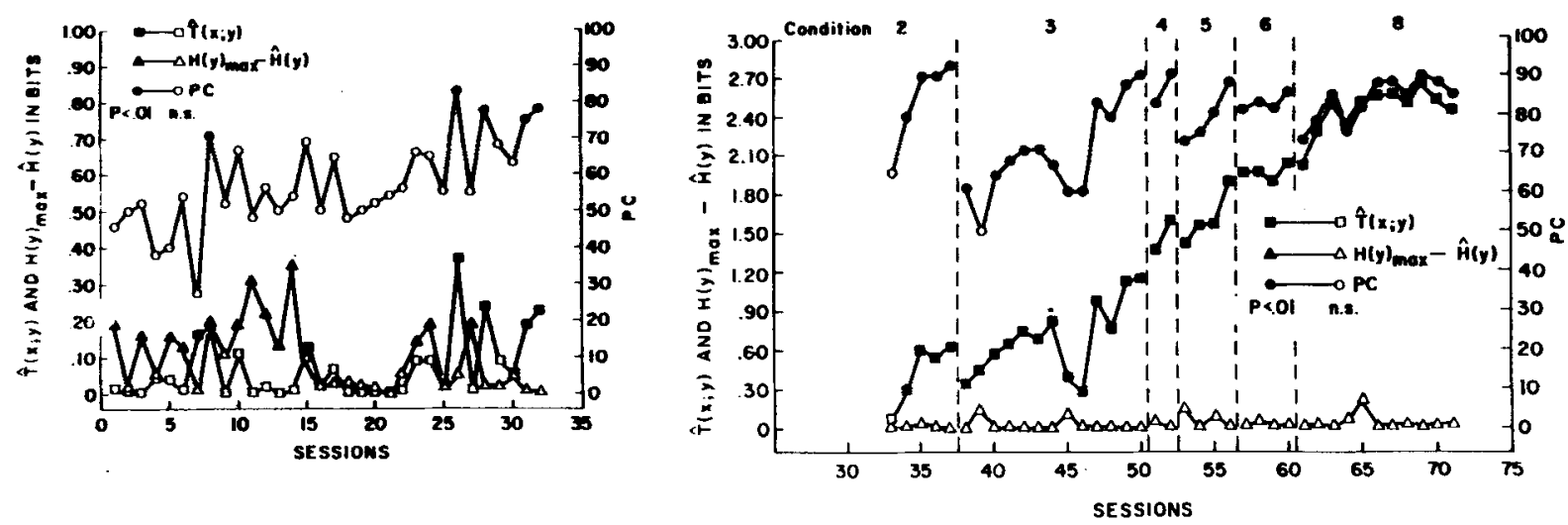

Fig. 6. The amount of information about the sample stimulus transmitted by the responses, $T(x ; y)$; the position preference, $H(y)_{\max }-\hat{H}(y)$; and the percentage of correct responses, PC, in each training session for S 14. (A) Condition 1 . (B) Conditions 2-8.

The rapid rate of acquisition of additional comparison stimuli indicates that the extra training required to match with eight comparison stimuli was warranted, since eight comparison stimuli yield three times the information obtained with only two. The high level of performance displayed in Condition 8-reversed indicates that the birds comprehended the matching task and were not acting on the basis of position or other extraneous variables.

There was no evidence that the training in Condition $2 b$ (all adjacent pairs of stimuli) improved the performance of $\mathrm{S} 11$ in the subsequent conditions. However, more work will have to be done to determine optimum training procedures.

In contrast with $\mathrm{PC}, \mathrm{T}(\mathrm{x} ; \mathrm{y})$ indicated the difficulty of each condition as well as the level of performance. $\mathrm{H}(\mathrm{y})_{\max }-\hat{\mathrm{H}}(\mathrm{y})$ was useful in demonstrating position preference, particularly in the early sessions.

The success of this study demonstrates that the simultaneous matching-to-sample procedure with more than two comparison stimuli is an efficient means of obtaining information about the discrimination capacity of the pigeon. Presumably, the technique could be varied so that it would be suitable for other species with well-developed visual systems, particularly monkeys. For some applications, it may also be an expedient technique for use with humans.

In order to use the matching-to-sample procedure

Table 2

Performance on Test Conditions

\begin{tabular}{lccccccc} 
& & \multicolumn{3}{c}{ T(x;y) in Bits* } & & \multicolumn{3}{c}{ PC } \\
\cline { 2 - 4 } \cline { 6 - 8 } $\begin{array}{c}\text { Test } \\
\text { Condition }\end{array}$ & 11 & $\begin{array}{c}\text { Subjects } \\
1.2\end{array}$ & 14 & & 11 & 12 & 14 \\
\hline 2 & .91 & .91 & .75 & 99 & 99 & 96 \\
4 & 1.68 & 1.79 & 1.75 & 93 & 96 & 94 \\
6 & 2.30 & 2.29 & 2.37 & 94 & 93 & 96 \\
8 & 2.59 & 2.58 & 2.77 & 90 & 90 & 94 \\
8 8-Reversed & 2.71 & 2.38 & 2.63 & 93 & 83 & 91 \\
\hline
\end{tabular}

${ }^{*} T(x ; y)-T h e$ amount of information about the sample stimulus transmitted by the responses.

${ }^{*} P C-$ Percent correct. effectively to study perceptual and conceptua organization, as discussed earlier, a broader range o matching ability must still be demonstrated. First, i must be shown that pigeons can make a dimensiona matching response rather than an identity matchin response, i.e., it must be demonstrated that pigeons cal match on the basis of some dimension such as size o color when the sample and the appropriate compariso stimulus are different on other dimensions. With such matching procedure, the perception of a stimulus on : known dimension can be assessed. In order to assess th perception of a stimulus on more than one dimension, $i$ must also be shown that the pigeon can match to sampl when the dimension on which the comparison stimul vary changes between trials.

The results of this study indicate that such furthe investigation of the matching-to-sample procedure is warranted. To be practical, this will have to be dont with more automated equipment. The stimulus display windows can serve as response sensors, the piezoelectric pecking key described by Altman \& Hull (1973) being well suited for this purpose. Trials could be initiated by sensing with photocells when a $\mathrm{S}$ was in the appropriate location in the starting area.

Simultaneous matching to sample may provide a powerful method for the investigation of visual perception and concept formation in infrahuman Ss.

\section{REFERENCES}

Altman, F., \& Hull, L. D. Piezoelectric pecking key. Journal of the Experimental Analysis of Beahvior, 1973, 19, 289-291.

Attneave, F. Applications of information theory to psychology New York: Holt, 1959.

Cohen, L. R. Generalization during acquisition, extinction and transfer of matching with an adjustable comparison. Journal of the Experimental Analy sis of Behavior, 1969, 12, 463-474.

Mallot, R. W., \& Mallot, M. K. Perception and stimulus generalization in W. C. Stebbins (Ed.), Animal psychophysics. New York: Appleton-Century-Crofts, 1970.

Miller, G. A. The magical number seven plus or minus two: Some limits on our capacity for processing information. Psychological Review, 1956, 63, 81-97.

U.S. Department of Commerce, National Bureau of Standards. Tables of the binomial probability distribution. Washington. D.C: U.S. Goverment Printing Office, 1950.

(Received for publication May 1, 1973; revision received October 4,1973 .) 